\title{
Trente ans de luttes politiques et syndicales à Oaxaca au Mexique
}

\author{
Ethnographie des mémoires personnelles et \\ collectives \\ Julie Métais \\ (Doctorante EHESS / Université de Montréal)
}

\begin{abstract}
Résumé / Abstract
[Fr] Cet article s'attache à sonder les enjeux politiques du rapport au passé au sein d'un groupe très actif politiquement dans l'État d'Oaxaca au Mexique : la section locale du Syndicat national des travailleurs de l'éducation. Confontant les mémoires des luttes passées existant au sein de ce groupe, cet article aborde deux registres différenciés - individuel et collectif - selon lesquels le passé est mobilisé. Il montre comment ces mémoires, saisies de manière ethnographique, loin de s'entrechoquer, prennent sens une fois insérées dans leur contexte et nous donnent des éléments de compréhension de la vie politique de ce groupe.

Mots-clés : mémoire, Mexique, syndicat, méthode ethnographique.

[En] This paper examines the political importance of the past in a very active organization in the state of Oaxaca, Mexico: the local branch of the National Union of Education Workers. Confronting the memory of past mobilizations that exists at the center of this group, this analysis examines how history is engaged on both the individual and collective levels. Ethnographic research is used to show how these memories, when studied in their social and political context, can provide important insight into the political life of this organization.
\end{abstract}

Keywords: Memory, Mexico, Union, Ethnographic method.

\section{Introduction}

En 2010 dans l'État d'Oaxaca au Mexique, tandis que l'État mettait en scène à l'échelle nationale, de façon spectaculaire, le centenaire de la Révolution ainsi que le bi-centenaire de l'Indépendance du pays, la direction de la section locale oaxaqueña (de l'État de Oaxaca) du syndicat national des instituteurs et institutrices revendiquait « Trente ans de lutte magisterial ${ }^{\dagger}$ et populaire pour la démocratie ». Depuis la

${ }^{1}$ Du corps des professeurs d'école. 
fin des années 1970, cette section syndicale a effectivement joué un rôle majeur dans la vie politique locale. La section 22 a connu, durant toute la décennie des années 1980, un processus de démocratisation syndicale qui n'a connu son pareil au Mexique qu'au Chiapas. Plus récemment en 2006, le syndicat des instituteurs a joué un rôle important d'animation et de mobilisation de la population de Oaxaca lors de l'épisode contestataire de la $\mathrm{APPO}^{2}$, de juin à novembre 2006. Fort impliqués au sein de l'APPO, les instituteurs de la section 22 ont enduré une répression sévère de la part des polices locale puis fédérale. Le mouvement de contestation du gouvernement local avait débuté suite à une violente intervention dans l'espace public de la police locale, visant ces instituteurs. Ceux-ci avaient été délogés du Zócalo (place centrale) de la ville qu'ils occupaient comme chaque année afin de négocier avec le gouvernement local de meilleures conditions de travail ainsi qu'un certain nombre de revendications salariales.

Présente à Oaxaca en 2010 dans le cadre de ma recherche, j'ai pu assister à de nombreuses cérémonies commémoratives d'épisodes politiques relevant tant de l'histoire locale que nationale. Par exemple, chaque année depuis 2006, la section 22 commémore les dates anniversaires de la répression dont le mouvement a été victime à l'occasion de rassemblements dans la capitale. Tous les ans, le 14 juin, date en 2006 de l'entrée de la police locale sur le Zócalo de la ville de Oaxaca pour déloger les instituteurs de leur plantón (occupation politique de l'espace public), une grande manifestation à travers les rues de la capitale réunit ainsi une partie des instituteurs de l'État ainsi que des membres d'organisations sociales et politiques locales ayant participé à la APPO. L'année 2010 correspondait aussi à une double célébration : celle du bicentenaire de l'Indépendance ainsi que du centenaire de la Révolution - deux événements majeurs de l'histoire politique officielle de ce pays. À l'occasion de ces différentes célébrations et commémorations, instituteurs, responsables syndicaux, militants locaux et pouvoirs publics ont pu donner aux publics présents - habitants, touristes - leur version de ces épisodes. Entre contestation et nécessité de construire une image valorisée de la nation, une série d'usages politiques de l'histoire ont alors été mis en œuvre. Parallèlement, lors de discussions plus informelles, $\mathrm{j}$ 'ai pu appréhender des formes plus subjectives et individuelles de cette histoire politique. C'est une partie de ce matériau, recueilli lors d'une enquête menée à Oaxaca en 2010, que j'aimerais analyser ici, en questionnant les enjeux politiques du rapport au passé dans un tel contexte, puisque, tel que l'énoncent Hartog et Revel (2001) : « les pouvoirs, les institutions, mais aussi tout un chacun ont la tentation récurrente de mobiliser les ressources cognitives, argumentatives, symboliques du passé ». Entre commémoration et témoignage, mémoire collective et mé-

2 Assemblée populaire des peuples de Oaxaca (Asamblea Popular de los Pueblos de Oaxaca). 
moire individuelle se donnent à lire des convocations du passé régies par des régimes variés.

La mémoire de cette histoire politique locale devient ainsi corpus mouvant fait de régularités et de distinctions. Dans la préface de son ouvrage Les lieux de mémoire, Pierre Nora souligne la dimension politique de la mémoire, entendue comme «un jeu de forces qui transforment la réalité ». Il précise encore que la mémoire est «un cadre plus qu'un contenu, un enjeu toujours disponible, un ensemble de stratégies, un être-là qui vaut moins parce qu'il est que par ce que l'on en fait » (Nora 1984 : VIII). Par ces mots, l'auteur insiste sur la dimension tant stratégique que fluctuante de la mobilisation du passé. J'aimerais pour ma part montrer de quelle façon cette variabilité des mémoires individuelles et collectives constitue non pas un obstacle heuristique mais bien au contraire l'opportunité d'appréhender la complexité des mondes sociaux. La nature des différents registres mobilisés pour évoquer, célébrer le passé, insérés dans leurs contextes d'énonciation, sont ainsi autant d'indices et d'éléments pour mesurer les enjeux à l'œuvre dans la convocation de ce passé. Assayag, commentant les travaux de Geertz, écrit :

« [Qu'] il n'y a pas une histoire de quiconque avec un seul visage ou un unique portrait, ni même une biographie, mais bien une profusion d'histoires, une nuée de biographies, une conjonction d'événements qui (se) sont passés. On ne saisit jamais que des bribes d'existence, au mieux rassemblées sous la forme de descriptions, plus ou moins pénétrantes ou contextualisées, mais toujours façonnées - "après les faits" » (Assayag $2007: 233$ ).

C'est donc à partir de ces bribes d'histoire et de multiples expériences que je propose de travailler. Me référant à nouveau aux travaux pionniers de Nora en la matière, je partirai d'une approche de la mémoire énoncée comme suit :

«La mémoire est la vie, toujours portée par des groupes vivants et à ce titre, elle est en évolution permanente, ouverte à la dialectique du souvenir et de l'amnésie, inconsciente de ses déformations successives, vulnérable à toutes les utilisations et manipulations, susceptible de longues latences et de soudaines revitalisations » (Nora 1984 : XIX).

Quels sont les liens établis dans le temps et dans l'espace par ces « histoires racontées », ces témoignages et ces célébrations ? Selon quelles modalités actualisentelles, réinventent-elles le passé ? Quels choix et sélections sont effectués, dans quels contextes? Pour aborder ces questions touchant au rapport au passé au sein d'un groupe syndical, je propose de croiser histoires officielles et intimes insérées dans leur cadre d'énonciation. Pour cet article, je ferai dialoguer une situation formelle et officielle de célébration d'un anniversaire de la vie politique oaxaqueña, orchestrée par la direction syndicale de la section locale du SNTE, avec le récit intime d'une expérience de militance émanant d'un instituteur aujourd'hui retraité. De cette façon, j'aborderai les enjeux politiques afférents à la mobilisation du passé, les liens établis dans le temps et dans l'espace par ces exercices différenciés de la remémoration ainsi que les dimensions d'actualisation, voire de réinvention du passé. 


\section{Contexte et enjeux politiques de la remémoration et de la célébration des luttes syndicales menées par les institutrices et instituteurs}

Traiter de la mémoire dans le contexte oaxaqueño nécessite dans un premier temps d'inscrire l'analyse dans le temps et de prendre en compte les dynamiques politiques locale et nationale. Cette réflexion prend place en effet dans un contexte national de «transition démocratique »- dont la portée semble réduite si l'on considère les possibilités concrètes d'expression politique et la permanence de politiques répressives (Nuijten 2004) ${ }^{3}$. Localement, il s'agit de considérer tant la spécificité de l'histoire politique locale que la place particulière qu'occupent les institutrices et instituteurs de cette région au sein de l'espace politique local.

\subsection{La vie politique oaxaqueña}

L'État d'Oaxaca a connu depuis le $19^{\text {ème }}$ siècle de nombreuses périodes de conflits politiques opposant la population aux gouverneurs locaux. Différentes mobilisations ont ainsi entraîné la chute des gouverneurs Edmundo Sanchez Cano en 1947, de Manuel Mayoral Heredia en 1952 et de Manuel Zárate Aquino en 1977. Suite à la violente répression du mouvement étudiant à Mexico en octobre 1968, des fronts ou coalitions rassemblant des étudiants, des paysans et des ouvriers se sont organisé partout dans le pays autour de revendications communes. À Oaxaca dans les années 1970-1980, la COCEI $^{4}$ et la COCEO $^{5}$ relevaient de telles expériences de coalition, occupant la scène politique locale, innovant dans les formes d'action collective et de contestation du système politique priista ${ }^{6}$. Plus récemment, la région a connu à nouveau des conflits opposant une partie de la population au gouvernement local, donnant lieu à des épisodes de répression sévère : en 2006, le mouvement de l'APPO $^{7}$,

\footnotetext{
${ }^{3}$ En effet, malgré l'annonce d'un processus de démocratisation, le déclin du PRI et un changement formel de régime en 2000 avec l'accession de Vicente Fox du PAN à la présidence, il subsiste une coexistence paradoxale entre pratiques de corruption, violence d'État ${ }^{3}$ et l'affirmation continue de valeurs démocratiques par les équipes politiques au pouvoir. Les réalités des relations de pouvoir entrent de ce fait en contradiction avec une idéologie nationale de démocratisation proclamée par l'appareil d'État (Lomnitz-Adler 2001 : 82).

${ }^{4}$ Coalición Obrero Campesina Estudiantíl del Istmo : Coalition ouvrière paysanne étudiante de l'Isthme.

${ }^{5}$ Coalición Obrero Campesina Estudiantíl de Oaxaca : Coalition ouvrière paysanne étudiante de Oaxaca.

${ }^{6}$ Du PRI (Partido Revolucionar Institucional).

7 Assemblée populaire des peuples d'Oaxaca.
} 
regroupant un ensemble d'organisations politiques, syndicales, citoyennes et d'habitants au sein d'une assemblée, s'est opposé frontalement durant plusieurs mois au gouvernement local. L'APPO a été créée le 19 juin 2006, dans un premier temps pour soutenir les enseignants en grève de la Section 22 qui occupaient le zócalo. En juin 2006, le gouverneur Úlises Ruiz Órtiz les a faits déloger par la police. La violence déployée à cette occasion a suscité la formation d'un mouvement de solidarité envers les professeurs qui demandait la destitution du gouverneur. L'APPO a été progressivement rejointe par une multitude d'organisations indigènes, associatives, ONG, ainsi que par des milliers d'habitants. Le mouvement a réuni plusieurs mois durant une partie considérable de la société locale. De juin à novembre 2006, le syndicat des instituteurs a joué au sein de cet espace un rôle déterminant - parfois contesté - de mobilisation et d'animation du processus collectif. Il n'est pas aisé de saisir les enjeux sociaux et politiques d'un tel événement, notamment parce que, comme le rappelle Dobry $(2010$ : 66) : «confrontées à des «événements» critiques tels que les « révolutions», les «crises politiques» ou les «transitions », les sciences sociales sont en permanence exposées à ce que j'ai appelé l'illusion hérö̈que (Dobry 1983) ». Quelques années plus tard, en 2010, outre les célébrations nationales du bicentenaire de l'Indépendance et du centenaire de la Révolution dont les commémorations ont rythmé la vie publique en $2010^{8}$, l'agenda politique est venu rappeler de façon aigüe ces enjeux de la vie politique locale soulevés en 2006 - parmi eux la corruption et l'impunité des élites politiques, l'autoritarisme et la violence du gouvernement local priista ${ }^{9}$. En juillet 2010, les élections locales ont porté à la tête de l'État de Oaxaca Gabino Cué Monteagudo, issu de la coalition «Unis pour la paix et le progrès ${ }^{10}{ }^{10}$ actant le départ d'Ulises Ruiz Ortiz et du PRI, départ qui, exigé durant des mois ${ }^{11}$ par l'APPO, n'avait pu être obtenu. Le syndicat des instituteurs, qui affiche une autonomie vis-à-vis des partis politiques ${ }^{12} \mathrm{n}$ 'a d'ailleurs pu soutenir ouvertement ce candidat d'opposition, tout en exhortant la population à aller voter de façon à faire tomber le « Tyran ».

\footnotetext{
${ }^{8}$ Une bonne partie de l'enquête a été menée en 2010, et les données ethnographiques mobilisées pour cet article ont été recueillies de mai à septembre 2010.

${ }_{9} \mathrm{Du}$ PRI : Parti révolutionnaire institutionnel : parti au pouvoir au Mexique durant près de 70 ans (jusqu'en 2000), dénoncé pour ses pratiques de corruption, de cooptation des élites syndicales. Ce parti est aujourd'hui de nouveau à la tête du pays suite à l'élection d'Enrique Peña Ñeto à la présidence, en juillet 2012 .

${ }^{10}$ Coalition hétéroclite de partis d'opposition au PRI réunissant le PAN (Parti d'action nationale), le PRD (Parti révolutionnaire démocratique), Convergencia (Convergence) et le PT (Parti des travailleurs).

${ }^{11}$ Notamment via la procédure menée auprès du Sénat de la République de mise en œuvre de la « disparition des pouvoirs ».

12 Autonomie qui est d'ailleurs inscrite dans les principes directeurs régissant le fonctionnement du syndicat.
} 
Autre front contemporain de la conflictualité politique oaxaqueña : la région est concernée par le Plan de développement économique et industriel Puebla Panama, initié en 2001, devenu depuis le Plan mésoamérique. Soutenu par la BID et la Banque mondiale, ce plan a pour but d'attirer les investisseurs étrangers dans la région pour la mise en œuvre de projets de développement dans différents domaines d'activité. Longtemps restés en suspens, aujourd'hui un certain nombre de ces projets ont été engagés dans l'État de Oaxaca avec notamment l'implantation depuis 2005 d'un parc éolien dans la région de l'Isthme de Tehuantepec, des sites d'extraction minière dans les Valles et la Sierra Norte et le projet de barrage hydroélectrique à Paso de la Reina. Ces projets sont contestés par les organisations locales paysannes et indiennes, qui dénoncent les pratiques d'intimidation des entreprises, le défaut de concertation et les impacts sociaux-environnementaux négatifs ${ }^{13}$ de ces réalisations. Bien souvent au sein des communautés concernées par ces projets, les instituteurs et institutrices jouent un rôle d'information, de relais et de mobilisation ; dans certains cas, ils se chargent de faire l'intermédiation entre ces villages et la Section 22 du syndicat afin qu'elle leur apporte un soutien logistique, humain, ou politique.

Enfin, ajoutons que suite à la libéralisation politique engagée depuis les années 1980, le pays connaît un contexte de fragilisation des corporatismes d'État. La remise en cause formelle des médiations traditionnelles opérées par les partis et les syndicats est souvent lue comme un facteur nécessaire à la démocratisation des pratiques politiques, marquant le passage progressif de corporatismes d'État à des systèmes pluralistes. Parallèlement, depuis quelques années, une partie du monde syndical national fait les frais d'une politique plus répressive de la part du gouvernement national ${ }^{14}$. Cette nouvelle donne des relations État-syndicat joue sur les répertoires d'action des syndicats, et particulièrement sur les registres du discours mobilisés par les représentants de la section locale du SNTE. La Section 22 a elle aussi connu diverses formes de répression de la part de l'État ; cependant les relations entretenues avec le gouvernement local perdurent par ailleurs selon un mode traditionnel de négociation-cooptation.

Voyons à présent la place politique spécifique occupée à Oaxaca par la section locale du SNTE.

\footnotetext{
${ }^{13}$ Source : La Jornada, octobre 2005.

${ }^{14}$ En témoigne l'extinction de Luzy Fuerza del Centro (Lumière et force du centre), organisme décentralisé responsable de la production, l'acheminement, la transformation, la distribution et l'approvisionnement de l'énergie électrique dans les États du centre du pays, relié au SME (Syndicat mexicain des électriciens), l'une des plus grandes centrale syndicale du pays. En mai 2009, des milliers de travailleurs syndiqués ont été congédiés par le Président Felipe Calderón, après que la police fédérale préventive (PFP) ait pris possession par la force des bureaux et installations de l'organisme.
} 


\subsection{Les instituteurs de l'État d'Oaxaca : entre radicalité et charrismo $^{15}$}

Au Mexique depuis les années 1940, les institutrices et instituteurs jouent un rôle politique d'importance souligné par de nombreux auteurs (Recondo 2002 ; Street 1997 ; Lomnitz 2001). Outre un rôle historique d'intermédiaires politiques entre l'État et les communautés, ils sont très présents dans l'espace public mexicain, particulièrement depuis les années 1980. À Oaxaca, chaque année depuis 1981, ils occupent durant plusieurs semaines le zócalo de la capitale de l'État. Parallèlement, ces derniers organisent régulièrement durant cette période des manifestations et blocages de routes afin de peser sur ces pourparlers.

La singularité de la place politique occupée aujourd'hui par ce syndicat à Oaxaca tient en partie au processus de démocratisation de la section locale du SNTE à la fin des années 70, qui a donné naissance en 1979 à la Section 22, reliée à la $\mathrm{CNTE}^{16}$. Ce processus marque un moment important de transformation du rapport syndical des maestros et maestras ${ }^{17}$ de l'Oaxaca à l'État central ${ }^{18}$. Ce mouvement de démocratisation locale n'a connu son pareil qu'au Chiapas, il visait à mettre en place un fonctionnement syndical plus horizontal, démocratique et transparent. Les éléments du nouveau fonctionnement interne démocratisé de la section locale ont alors été fixés dans le cadre des Veinte principios rectores ${ }^{19}$ aujourd'hui encore en vigueur. Ce courant syndical démocratique oaxaqueño s'est opposé durant toutes les années 1980 à une frange plus conservatrice du syndicat, la Vanguardia Revolucionaria ${ }^{20}$, jusqu'alors hégémonique au sein du SNTE, appuyée par les gouvernements local et fédéral. Ce courant de la Sección 22 est aujourd'hui largement majoritaire. Il a constitué une forme d'apprentissage politique pour ces derniers, tel que le décrit Rubin :

« This organisation fought to establish democratic procedures for leadership selection and for the union's exercise of power within the educational system, procedures that challenged the union's corrupt leadership. By encouraging local teachers to join together and fight abuses of power locally, as well as press for representative union congresses at the state and national levels, the democratic teachers' movement served as an education ground for teachers in political discussion and mobilisation, as well as internal democracy. The movement

\footnotetext{
15 Désigne au Mexique les pratiques d'alliances entre leaders syndicaux et appareil gouvernemental ou organisations patronales.

16 Coordinadora Nacional de los Trabajadores de la Educación : Coordination Nationale des Travailleurs de l'Education, qui réunit l'ensemble des sections revendiquant un fonctionnement démocratique.

17 Instituteurs et institutrices.

18 L'histoire de cette organisation a fait l'objet d'une série de travaux menés par Zafra, Martinez Yescas (1985) et Martinez-Vásquez (1990).

19 Principes directeurs.

${ }^{20}$ L'Avant-garde Révolutionnaire.
} 
resulted from a combination of grassroots organising among teachers and conflicts within the central state regarding the strength of the union leadership, and it included PRI supporters as well as supporters of opposition parties. In this way, tensions within the state, with their own historical origins, combined with the politicisation of people's work experiences to produce an explicitly oppositional organisation, as well as alternative beliefs and practices regarding democracy ». (Rubin $2001:$ 127)

Cette démocratisation a permis aux maestros oaxaqueños de contester les abus du pouvoir local, de demander une représentativité des congrès syndicaux au niveau local et national (Recondo 2002). En dépit de ce processus de démocratisation syndicale, les analystes du fait politique oaxaqueño observent depuis les années 1990 le retour en force de pratiques syndicales plus classiques d'organisation verticale, de cooptation, de corruption des élites syndicales (Zafra \& Raul-Yescas 2006). En 2006 par exemple, le dirigeant de la Section 22 Enrique Rueda Pacheco avait dû quitter la région durant le mouvement de l'APPO suite à la publicisation de ses pratiques corrompues. En outre, partout dans l'État, les parents d'élèves dénoncent les absences répétées des instituteurs s'éloignant des communautés pour participer aux activités syndicales. Critiqué, dénoncé en interne tout comme par la société locale, le syndicat oaxaqueño et son corps de professeurs souffre aujourd'hui d'un manque de légitimité.

Ce contexte social, historique et politique ne peut qu'influer sur les pratiques de témoignage, de remémoration et de commémoration du passé. Quelques années après un mouvement de contestation qui a ébranlé tout l'État, alors que des manifestations ont lieu régulièrement pour demander justice et réparation suite aux violences occasionnées par la répression d'État, il est essentiel de lire les mémoires, leurs registres et les stratégies afférentes à la lumière de ce contexte politique local. Il convient de questionner le régime d'historicité dans lequel les questions se posent, notamment s'agissant du rapport revendiqué et complexe de ce syndicat à un processus controversé de démocratisation et d'évolution des règles du jeu politique au Mexique. Ce syndicat qui, via sa composante « démocratique », la CNTE, a conçu sa mobilisation pour la démocratisation durant les années 1980 comme touchant simultanément trois champs intimement liés : le syndicat, l'école et le pays. Enfin, en 2010, la direction syndicale prépare pour l'année suivante les célébrations de «trente ans de lutte et de mobilisation pour la démocratie ». Il est par conséquent délicat d'appréhender le rapport au passé de ce groupe socio-professionnel sans tenir compte des multiples sollicitations et implications politiques travaillant au présent ces pratiques de commémoration et de remémoration. Parmi ces dernières se trouvent donc les enjeux électoraux, la crise de légitimité du syndicat, l'impunité dont jouissent les élites politiques, mais encore plus largement les héritages complexes de cette Révolution célébrée en 2010. 
Suivant une méthode ethnographique, à l'instar de l'historienne Kay Vaughan, je considère qu'il importe pour une démarche historique de lier approches macro et micro de façon à lier une ethnographie locale à des phénomènes économiques, politiques et culturels plus larges ${ }^{21}$. J'aborderai donc dans la partie qui suit un matériau ethnographique saisi au niveau micro-sociologique, qu'il convient d'analyser en tenant compte des éléments de contexte plus généraux précédemment posés. C'est à cette condition, selon un aller-retour nécessaire entre données contextuelles et récits ethnographiques, que l'on peut donner sens aux différentes façons de mobiliser le passé.

\section{Mémoires à plusieurs voix : l'instituteur, le cadre syndical et l'archiviste}

J'ai choisi de sonder les enjeux, sens et effets de la mobilisation des mémoires de luttes politiques émanant de ces instituteurs syndicalisés depuis différents espaces. Un espace public tout d'abord, où lors de la commémoration d'une journée de répression en 2006, une parole syndicale officielle est mise en scène, exaltant l'histoire du mouvement syndical et «populaire ». J'aborderai également de façon complémentaire les enjeux relevant d'une initiative de mise en archives de l'histoire du syndicat portée au sein de la Section 22 ; ces deux aspects s'appuieront sur les notes de mon carnet de terrain, matériau ethnographique dont j'extrairai des séquences.

Ensuite, j'ai souhaité confronter ce matériau à la parole exprimée depuis un espace intime, soit un extrait de journal de militance lu et commenté par un instituteur retraité de la Section 22. Ce témoignage singulier m'intéresse au-delà de toute représentativité, je m'inspire en cela de la démarche casuistique de Passeron et Revel (2005), également défendue par Cefaï qui écrit que «l'ethnographie "pense par cas" qu'elle "raisonne à partir de singularités" qu'elle explore en profondeur et dont elle prend la mesure avant de, peut-être, proposer quelque généralisation » (Cefaï 2010 : 576).

\footnotetext{
21 « penetrating historical analysis comes through integrating macro and micro approaches linking local ethnography to larger political, economic and cultural phenomena. » (Kay Vaughan 2001: 485-486)
} 


\subsection{La célébration syndicale d'un épisode marquant de la vie politique et syndicale locale}

En juin 2010, j'ai pu assister à la mise en scène de la commémoration par le syndicat oaxaqueño du violent desalojamiento ${ }^{22}$ des instituteurs qui a eu lieu le 14 juin 2006 alors que ces derniers occupaient le zócalo de Oaxaca. À cette date, au sein du syndicat, partout des critiques s'élevaient, contestant le retour en force des pratiques de décision autoritaires et verticales sans prise en compte de ceux que la direction de la Section 22 désigne comme «las bases $»^{23}$. Par ailleurs, la relation entre les instituteurs et les parents d'élèves n'a fait que dégénérer depuis 2006 dans la région, ces derniers leur reprochant leurs nombreuses absences et leur peu d'implication pédagogique et communautaire. Enfin, après quatre ans, le crédit gagné en 2006 par les professeurs de la Section 22 au sein de l'APPO commençait à s'estomper, tandis que le rôle politique et les objectifs du syndicat, les intérêts, stratégies des dirigeants étaient partout soumis à la critique. C'est dans ce contexte qu'il faut lire cette célébration commémorative, à un moment où le syndicat manque de légitimité, sa direction et son personnel sont accusés de pratiques corrompues et de ne pas garantir la mission éducative du corps de professeurs. Je mets donc en œuvre dans la partie qui suit une ébauche d'ethnographie des formes instituées de la mémorialisation, telle qu'a pu la proposer par exemple Abélès (1986 \& 1989). J'ai observé et consigné un ensemble de notes lors de mon terrain sur ce que l'on peut désigner comme un « dispositif rituel » qui se répète chaque année à Oaxaca depuis 2006, par lequel la direction syndicale se donne à voir sur la place centrale de la ville tant aux pouvoirs locaux qu'à la société oaxaqueña, aux touristes nationaux et internationaux de passage. Je choisis de ne pas segmenter cet épisode, mais bien plutôt de le présenter dans sa continuité sur la base de mes notes de terrain, afin de l'analyser dans sa totalité, tel un rituel aux contours délimités dans le temps. Symboles et rituels ponctuent la vie politique syndicale oaxaqueña et prennent une place déterminante, ils méritent d'être analysés comme éléments de la perpétuation du rapport au passé (Fassin 2008 $: 176)$.

Extrait d'un carnet de terrain daté du 14 juin 2010, jour anniversaire de la répression des institutrices et instituteurs :

«C'est la journée de la Mégamarcha ${ }^{24}$ des institutrices et instituteurs de tout l'État d'Oaxaca, journée de mobilisation générale pour rappeler la répression de 2006 et célébrer le mouvement contestataire de l'APPO, auquel la Section 22 a largement pris part. Nous rejoignons le cortège, il est impressionnant, ressemble

\footnotetext{
${ }^{22}$ Intervention policière musclée pour faire place nette sur cette place et mettre fin au campement des instituteurs.

23 « Les bases » du mouvement.

24 Très grande manifestation.
} 
à une marée humaine. Globalement ce cortège est très calme, avec relativement peu de consignes et chacun marche de façon disciplinée. Je suis surprise de noter qu'il n'y ait presque aucune organisation sociale ou politique autre que le syndicat présente à l'occasion de cette manifestation en mémoire du 14 juin 2006.

Au fur et à mesure que le cortège se rapproche du zócalo, des professeurs affluent et le cortège enfle. Depuis le kiosque occupant en hauteur le centre de la place une bonne partie de la direction locale du syndicat est réunie pour le meeting habituel de fin de manifestation. Le Secrétaire Général prend la parole ${ }^{25}$ au micro : «Vengeance à qui a réprimé, à qui a puni, à qui a assassiné le peuple de Oaxaca. À ces veufs, à ces orphelins, à ces hommes, qui n'oublient pas qu'un gouvernement maladroit a agressé un mouvement social, à l'APPO. Aujourd'hui, 14 juin 2010, grâce à cette manifestation, nous avons démontré, à nouveau, que le mouvement magisterial et le mouvement social sont forts et consolidés, organisés. Parce qu'ils ont des principes, parce qu'ils ont une histoire. Et cette histoire, dans le cas du mouvement magisterial, a été actualisée durant trente ans. En ceci, nos vieux instituteurs, quand ils ont créé les principes directeurs, ne se sont pas trompés quand ils ont dit que ce mouvement est un mouvement engagé auprès des peuples, c'est un mouvement de masses. C'est un mouvement libre dans son idéologie. Mais c'est une pensée qui s'appuie sur les prises décisions des compagnons de la base. Et, comme si ce n'était pas assez, récemment, de nouveaux principes se sont ajoutés à nos principes directeurs : celui de la défense de la souveraineté de nos peuples, comme l'égalité de genre, comme la défense de nos ressources naturelles. En cette année 2010, il y a de nombreuses raisons de lutter, au niveau local et national, de sortir dans la rue pour défendre ce à quoi l'on tient. Dans les communautés, et dans nos écoles. Afin de participer, de toutes les façons possibles, avec un peuple qui n'en peut plus de la soumission, un peuple qui demande justice. C'est pourquoi la Journée de lutte du mouvement magisterial, qui a commencé l'an passé avec la consigne : pour l'éducation, pour la justice, contre le terrorisme d'État. Chaque jour il y a des assassinats dans ce pays (...) Qui alors va hausser la voix contre ces attaques, contre ces militaires dans notre État et dans notre pays ? Contre les groupes paramilitaires derrière qui se trouvent les gouvernements fédéraux et locaux, avec une politique qui ne sert qu'à tromper les gens, à les maintenir soumis, enfermés en prison, avec la peur ? C'est pourquoi, maestras et maestros, aujourd'hui, il nous faut dire que la lutte continue. Aujourd'hui est un moment historique significatif, et nous devons chacun mettre notre grain de sable depuis nos terrains de luttes. Nous avons aussi poursuivi le mouvement magisterial et nous devons dire que, pour ceux pour qui ce n'est pas clair encore, pour ceux qui pensent que notre conscience, notre esprit n'est pas en accord avec les principes historiques de ce mouvement ».

${ }^{25}$ Traduction de l'auteure sur la base d'un enregistrement fait sur le moment. 
Pour ma part, j'observe le meeting assise sur un banc sur la place à proximité du kiosque, entourée d'institutrices et instituteurs venus «participer $»^{26}$. À quelques mètres seulement du kiosque, l'assistance est relativement indifférente aux propos tenus : les femmes maestras présentes tricotent et crochètent, tous autour de moi discutent entre eux en prêtant peu d'attention à ce qui est dit en tribune par le Secrétaire Général. Quant aux personnes de passage sur la place -habitants et touristes-, elles ne prêtent presqu'aucune attention à ce qui se passe en tribune et passent leur chemin comme si de rien n'était ${ }^{27}$. Aux abords immédiats du kiosque en revanche, les fidèles du secrétaire sont réunis, en tout quelques dizaines de personnes. Dans cet espace restreint chacun applaudit et chante avec ferveur, en levant le poing gauche quand le meeting s'achève avec la chanson Venceremos ${ }^{28}$, reprise en choeur. Ce chant clôt depuis trente ans la plupart des activités syndicales de la Section 22, qu'il s'agisse des meetings, des assemblées ou des manifestations ».

Le jour de cette date anniversaire du 14 juin 2006, le secrétaire général valorise ainsi le rôle historique du syndicat des professeurs à Oaxaca, fort de «Trente ans de luttes magisteriales et populaires pour la démocratie ». Il renvoie pour ce faire aux principes originels du mouvement de la fin des années 1970-début des années 1980, principes d'horizontalité, de transparence, et de proximité vis-à-vis des peuples de Oaxaca. Ces principes ainsi que la «grammaire politique » structurant le discours de ce dirigeant syndical renvoient à un processus de cadrage au sein de l'organisation syndicale que Cefaï définit comme « une praxis collective, dans des contextes d'interactions entre individus, entre réseaux et entre organisations, recourant à des répertoires d'identification, de narration et d'argumentation, à des réserves de savoir ou à des gisements d'expériences » (Cefaï 2001 : 98).

Deux repères temporels valorisant le rôle politique du syndicat structurent cette prise de parole : les origines « combatives » de ce dernier dans les années 1980, puis le mouvement de 2006 durant lequel les professeurs de la Section 22 ont joué un rôle essentiel, bien connu de la population locale. Ce discours commémoratif permet donc de saisir les liens intrinsèques qui sont faits entre des mobilisations menées à des époques différentes, mobilisations pour la démocratisation de la vie politique locale. Il s'agit là d'une mémoire officielle du syndicat, travaillée et structurée dans un

26 À noter, la participation syndicale au sein de la Section 22 est encouragée par un système de pointage de la présence de chacun à l'occasion des activités politiques organisées. Ce décompte peut, en fonction de la délégation à laquelle appartient l'institutrice ou l'instituteur, jouer sur l'obtention de crédits ou la possibilité d'une mutation.

${ }^{27}$ Ceci s'explique en partie par le fait que ce zócalo de Oaxaca est très régulièrement investi par les différents groupes mobilisés, syndicats, représentants de communautés en conflits installés sous des tentes, distribuant des tract ; ces occupations relèvent aujourd'hui dans la région d'un répertoire d'action collective devenu quasiment routinier auquel les passants ne prêtent plus guère d'attention.

${ }^{28}$ Chant de campagne de Salvador Allende au Chili, exaltant la lutte populaire pour le socialisme. 
contexte de nécessaire relégitimation d'un mouvement en perte de vitesse. Cette performance ritualisée -chanter l'hymne de campagne de Salvador Allende, Venceremos, en levant le poing gauche en est un élément-clé- contraste cependant avec la réception mitigée du public présent sur la place. Ce rituel commémoratif, dont certains éléments demeurent inchangés depuis des années, réactualisant dans le présent la période héroïque des débuts de la Section 22 et l'expérience de l'APPO, revêt désormais une tournure routinière en réaffirmant la fidélité à l'esprit contestataire et démocratique des origines. Dans ce cas, la mémoire apparaît comme ressource symbolique pour un groupe politique revendiquant l'incarnation de la résistance à un État autoritaire. Ce dirigeant syndical réactualise donc le passé proche et lointain pour se poser en héritiers d'une époque héroïque, forme d'historicisme. Cela donne des éléments de compréhension des ponts établis par cette organisation entre différents épisodes de l'histoire politique de ce groupe. En ce sens, l'ethnographie, comme l'écrit Abélès, permet d'envisager l'événement comme " point de départ pour mieux comprendre la cohérence et la signification de phénomènes qui peuvent appartenir à des époques différentes » (Abélès 1989 : 13). Cependant dans un tel contexte, cette commémoration semble avoir une efficacité performative limitée.

\subsection{L'archiviste ou le dépositaire jaloux de la mémoire du groupe}

À la même époque, la direction du syndicat a alloué des moyens humains et financiers considérables pour collecter, mettre en ordre et valoriser un ensemble d'archives ${ }^{29}$ témoignant des luttes menées par le syndicat depuis trente ans, dans le cadre du projet «Archiver et documenter trente ans de luttes syndicales et populaires pour la démocratie ». La personne en charge de ce projet bénéficie, depuis qu'elle a pris la direction de ce projet, d'une reconnaissance accrue de ses pairs au sein du syndicat, incarnant personnellement la mémoire de ces luttes. Elle est souvent appelée «archives sur pattes », en tant que dépositaire de la mémoire des années épiques de la lutte des instituteurs et institutrices de l'Oaxaca. Ayant été mise en contact avec cet archiviste, j'avais eu un premier rendez-vous avec lui, lors duquel il m'avait proposé la mise à disposition d'une partie de ces archives pour mon travail de recherche. Il m'avait également laissé espérer la possibilité d'une collaboration dans le cadre d'un projet de recueil filmé de témoignages d'instituteurs ayant participé au mouvement de démocratisation syndicale durant les années 1980. Cependant par la suite, et malgré mon insistance, je n'ai jamais pu poursuivre

\footnotetext{
${ }^{29}$ Ces archives, auxquelles j'ai eu accès par la suite, comprennent essentiellement des photos, tracts, compte-rendus de réunions, affiches, enregistrements et vidéos d'assemblées
} 
l'échange avec l' «archiviste », mais plus encore, j'ai trouvé le local où se trouvaient ces archives fermé à clé : la clé donnant accès à cette pièce ayant été « égarée ».

Ce décalage entre une volonté publique de publicisation de l'histoire «épique » des luttes menées par le groupe et les réticences de l'archiviste à me faciliter l'accès aux sources matérielles de cette mémoire syndicale est intéressant à analyser, à plusieurs titres. D'une part, il met en évidence que la mémoire et les traces du passé dans le présent ont une valeur, constituent une ressource précieuse, source de pouvoir au sein d'un groupe donné, de ce fait difficile à partager avec une personne extérieure. Cet archiviste s'était ainsi imposé au sein du syndicat -pour le moins auprès de la direction qui a accepté de financer le projet- comme le dépositaire de la mémoire du groupe. Son surnom «d'archives sur pattes» est intéressant dans le sens où il révèle à quel point cette personne a réussi à incarner cette mémoire du groupe. D'autre part, on saisit également qu'au regard de ce passé comme ressource pour le groupe syndical, il convient pour l'organisation d'avoir une prise sur la formulation, la valorisation publique de celui-ci, sur les choix à effectuer, les tris inévitables et certainement stratégiques à faire au moment d'une mise en récit. C'est pourquoi, tout en affirmant vouloir contribuer à une démarche de recherche sur le groupe, il était également difficile de me laisser avoir accès sans réserve à ces archives que j'aurais pu utiliser, interpréter à ma guise par la suite pour mes travaux. Or, il était possible que je formule une version non pas forcément critique mais différente de celle, épique et valorisante, construite par le groupe. Cet archiviste s'est de ce fait trouvé pris dans une situation de double-bind: représentant d'un syndicat démocratique prônant la transparence et les échanges critiques sur ses activités, il ne pouvait me refuser totalement l'accès à ces archives. D'un autre côté, ces cartons renfermaient les traces d'une histoire qu'il convenait d'explorer et d'exploiter tout d'abord en interne de façon à en tirer les éléments d'un récit conforme à cette lecture héroïque et épique du passé de l'organisation.

L'épisode commémoratif «officiel» précédemment présenté ainsi que ces réflexions tirées de mes échanges avec l'archiviste du syndicat méritent, pour prendre sens, d'être confrontés à d'autres mémoires des luttes, plus intimes et personnelles. Face à un récit autorisé tel que celui formulé en public par l'élite syndicale, quel peut être celui des individus ayant vécu ces événements et faisant acte de remémoration ? Ces récits autobiographiques sont-ils imprégnés des structures de cette mise en ordre officielle? Adoptent-ils des registres différents ? Leurs récits sont-ils jalonnés par les mêmes références ? Qu'est-ce qui fait sens au présent pour eux ? Je propose d'aborder ces questions en sondant un récit individuel et intime fort particulier : celui de F., instituteur à la retraite ayant exercé son activité professionnelle, syndicale à Oaxaca depuis les années 1970 et participé aux mobilisations pour la démocratisation de la section locale oaxaqueña du SNTE durant les années 1980. J'ai vu F. à de nombreuses reprises pour discuter de la Section 22 et de son implication passée au sein du syndicat. Après quelques rendez-vous, il m'a proposé de me 
faire partager certains extraits de son cahier de militance, sorte de journal sur lequel il prenait des notes lors de ses expériences de participation syndicale.

\subsection{Journal de militance d'un instituteur syndicaliste à la retraite}

Avant de donner à lire cet extrait de journal, quelques remarques méthodologiques et épistémologiques préalables sont nécessaires. Artières et Laé se sont penché sur les enjeux de l'exploitation historique de ce qu'ils ont appelé les «archives personnelles » (Artières \& Laé 2011). En introduction d'un ouvrage consacré à ce thème, ils posent une série de questions pertinentes pour appréhender également l'extrait que je vais utiliser :

«Que fait le scripteur en écrivant ce qu'il écrit et quels sont les destinataires implicites de chacune de ces archives ? Que dit-il et que fait-il lorsqu'il écrit ; que cherche-t-il à faire en écrivant pour lui et pour autrui, pour opérer quoi et se conduire comment ? À travers quelle contexture les auteurs se disent, s'exposent, s'engagent, se regardent en train de faire ou de penser faire ? En quoi ces écrits marquent les relations et les sentiments, le temps et l'action présents ; avec quel code et sur quel régime d'intensité ? Un autre point de vue pour saisir le social en acte ?» (Artières \& Laé $2011: 5$ ).

Ces auteurs évoquent une sorte d'interdit qui existerait au nom de la pudeur, de la protection de l'intime, comme s'il y avait « une frontière étanche et repérable entre l'individuel et le social » (Artières \& Laé 2011 : 6-7). Or, l'un des enjeux de leurs travaux est de montrer comment même les documents les plus intimes sont travaillés par le social et susceptibles d'en rendre compte également. Valorisant la teneur heuristique de documents tels que journaux, carnets de bord, etc... ces deux historiens défendent cette dimension micro-sociologique permettant une prise de distance avec l'histoire sociale classique essentiellement basée sur des matériaux publics agrégés : «En élargissant la notion d'écriture en sciences sociales, en donnant une place plus importante à la réflexivité, aux documents personnels dits privés, aux écrits de l'ordinaire, jusqu'alors peu visibles (et donc peu valorisés), on comprend l'idée de contre-épreuve : l'institution ne serait plus seule productrice d'archives » (Artières \& Laé 2011 : 10). La question de la représentativité d'un aussi petit échantillon se pose alors différemment pour aborder l'histoire sociale et des groupes, l'un des écueils demeurant «d'évacuer purement et simplement les dynamiques et le changement social » (Abélès 1996 : 100).

Cet extrait de journal m'a été lu et commenté par F. le 10 juin 2010 :

« Jeudi 24 avril $1997^{30}$ : nous sommes sortis à 6 heures du matin, la journée de marche devait être d'Acatlán à Tehuizingo, environ $43 \mathrm{~km}$. La sortie m'a causé

${ }^{30}$ Éléments traduits par l'auteur, sur la base d'un enregistrement et de notes prises sur le moment. 
une grande impression. Nous avons entonné deux hymnes de lutte: l'hymne national mexicain, et Venceremos. Le jour d'avant, je n'avais pas eu l'opportunité d'assister à cette émouvante sortie. (...)

Quand nous sommes sortis du village, la lune brillait et était resplendissante. Longtemps, nous avons marché protégés par ses beaux rayons. L'autoroute était entourée de terrains semés : certains avec du maïs déjà, d'autres préparés pour être semés. Comme tout ceci m'a rappelé ma terre natale, peut-être pour sa chaleur, et parce que c'était l'aurore. Parce qu'à ces mêmes dates quand j'étais enfant, très tôt le matin, nous allions, avec mon papa et mes frères, pour aller brûler les poubelles ou ramasser des mangues. Je garde en mémoire cette quiétude du matin. Cet air frais qui alimente les poumons, la lune qui disparaît peu à peu, perdant de sa luminosité pour laisser place à l'astre roi et tout ce qu'implique la symphonie sylvestre des petits oiseaux, les chants des coqs. (...)

Toute la foule humaine dévorant la bande d'asphalte est passée devant le regard des curieux; et la joie de ceux qui marchaient. Les gens se demandaient: «Et ceux-ci, qui sont-ils?

— Nous sommes les instituteurs, et nous exigeons des solutions ».

$F$. commente : "Et nous leur rappelions que l'instituteur qui marche assure aussi sa mission d'enseignement. Et même s'ils doutaient, partout où nous allions, nous criions : «Cette marche va arriver jusqu'au District Federal! ».

F. précise : «Ensuite nous sommes arrivés à Tehuizingo, puis nous sommes allés à Acatlán, pour arriver à Izucar de Matamoros, j'avais déjà les pieds recouverts d'ampoules. Je ne sais si tu vas me croire, mais presque toute la plante des pieds s'était décollée. La dernière portion du trajet, j'ai pensé que je n'allais pas pouvoir la marcher, parce que mes pieds me faisaient trop mal. Et pourtant, je n'ai pas voulu perdre l'opportunité d'aller en marchant. Certains montaient dans les camionnettes pour arriver mais... C'est pourquoi aussi à partir de ce moment j'ai arrêté d'écrire, à cause de la douleur que me causaient mes pieds. Je pouvais à peine marcher (...)».

Puis F. ajoute, à l'issue de sa lecture de cet extrait : « Mais non, l'impression que nous a laissée à tous cette expérience... Comme on se sent, quand les gens, les enfants sortaient à notre passage pour nous encourager, en criant... Ils nous donnaient à manger. C'est lors de ce mouvement que nous avons obtenu les livres d'école gratuits pour les collégiens ».

La séquence de journal choisie par cet instituteur à la retraite est un récit très intime d'une marche syndicale vers la capitale effectuée en 1997. Le choix par F. de cette séquence précise, fort éloignée des grands événements habituellement mis en valeur par la direction syndicale est marquant. Le ton personnel, le registre affectif, subjectif, émotionnel voire onirique de cet extrait est distinct de l'emphase héroïsante du discours du Secrétaire Général précédemment analysé. Cet extrait rend compte d'une expérience toute personnelle d'implication syndicale, d'une approche empirique d'activité politique, il donne à saisir avant tout l'expérience vécue et les émotions liées à la lutte : les souffrances endurées, ainsi que le rapport de solidarité existant avec les populations rencontrées et les gains sociaux de la lutte. F. retient et 
souligne par exemple les acquis liés à cet épisode de mobilisation : les livres d'école gratuits pour les collégiens de l'État de Oaxaca. Cependant, F. n'échappe pas à cette « grammaire syndicale » soulignée plus haut, il a recours aux grandes terminologies sur la combativité politique et la mission de transformation sociale de l'instituteur. En ce sens les attentes sociales des années 1980 envers les instituteurs ainsi que la grammaire syndicale transparaissent dans ces écrits de la subjectivité, ce que décrivent Artières et Laé : «Cette introspection est bordée d'inspections, suscitée par le halo d'interactions qui se mêle au discours, par des sentiments qui se sédimentent sur des attentes sociales, où se rejouent les effets de ces cadres » (Artières and Laé 2011 : 8). F., même lorsqu'il s'agit de ses écrits intimes, exprime une culture politique dont il est imprégné, culture politique entendue au sens de : "Opérations d'alignement des manières de percevoir, d'agir et de juger hétérogènes, d'articulation des modalités du vivre-ensemble dans des représentations de la collectivité, de justification ou de dénonciations des interventions dans l'espace public, de légitimation ou de critique de règles et d'usages de droit, d'échanges d'arguments sur le sens d'événements, de décisions ou d'action, de confections de biens communs et de biens publics » (Cefaï $2010: 99$ ).

Enfin, dans cet extrait, j'ai souhaité inclure les commentaires faits par F. lors de sa lecture de ce passage de son journal. Ceci permet de saisir de quelle façon ce dernier réévalue son expérience passé à la lumière du présent fait de corruption, de désenchantement et de perte du soutien de la part de la population. Par cette lecture commentée, F. a révélé une relation dialectique entre présent et passé inhérents à l'appréhension des processus mémoriels. Il a procédé à une actualisation du passé, ce passé qui lui semble aujourd'hui renié, bafoué. Cette liaison qu'effectue F. entre présent et passé renseigne autant sur le contemporain désenchanté que sur ce qu'a pu être l'expérience syndicale durant les années 1980-1990. Comme le souligne Bensa : «Les effets de sens sont alors rapportés aux liaisons que les personnes établissent d'elles-mêmes entre tous les événements, petits ou grands, passés et présents, qui adviennent »(Bensa 1996 : 65).

\section{Conclusion}

Ces deux cas sont édifiants pour ce qu'ils nous disent des aspects et du rôle de la mémoire dans la vie d'un groupe politique. Outre sa fonction instrumentale, il convient de repérer la variété des registres mobilisés, qui, au-delà du contenu discursif, prennent sens et portée au regard du contexte (historique et politique) d'énonciation. La scène du 14 juin 2010, confrontée à l'échange que j'ai eu avec F. au sujet d'un extrait de son journal permettent en partie d'illustrer la pluralité des mémoires coexistant au sein d'un groupe, entre aspects collectifs et individuels, intimes et stratégiques de la remémoration et de la mobilisation du passé. C'est ce que souligne Nora également quand il écrit : «Parce qu'elle est affective et magique, la mémoire 
ne s'accommode que des détails qui la confortent ; elle se nourrit de souvenirs flous, téléscopants, globaux ou flottants, particuliers ou symboliques, sensible à tous les transferts, écrans, censure ou projections » (1984: XIX). Un travail plus systématique d'analyse de ces «mémoires en actes » pourrait permettre d'identifier différentes «grammaires » de la mémoire, structurées selon des registres stratégiques, émotionnelles, mélancoliques, dont le contenu serait extrait d'un stock de mémoires qui, lui, revêtirait une dimension collective (Fentress \& Wickham 1994). Mises en contexte, ces mémoires variables intéressent la sociologie pour les sens sociaux dont elles sont porteuses une fois insérées dans leur contexte. Ainsi, tandis qu'une direction syndicale porte une mémoire officielle et formalisée - ou master narratives (Schmidt 2001) - du passé des luttes menées par le groupe, chaque individu porte lui une mémoire intime certes travaillée par ces master narratives, mais susceptible cependant d'adopter des registres différents, de mobiliser des expériences vécues, donnant à saisir des récits alternatifs aux histoires officielles mises en œuvre par cette direction syndicale ou par l'État. Le récit historique sort alors de son aspect lisse et linéaire. Plus encore, la confrontation de ces différentes grammaires de la mémoire, individuelles et collectives, nous donne les éléments nécessaires à tout effort de décentrement vis-à-vis de l'histoire sociale des groupes.

\section{Bibliographie}

Abélès, M. (1986). «L'Anthropologue et le politique ». L'Homme 97-98: 191-212.

Abélès, M. (1989). Jours tranquilles en 1989. Paris, Odile Jacob.

Abélès, M. (1996). «Le rationalisme à l'épreuve de l'analyse ». Jeux d'échelles. La micro-analyse à l'expérience. J. Revel. Paris, Seuil/Gallimard: 95-111.

Artières, P. \& J.-F. Laé (2011). Archives personnelles. Histoire, anthropologie et sociologie. Paris, Armand Colin.

Assayag J. (2007) Les politiques du savoir : histoire et anthropologie. Paris: Aux lieux d'être.

Bensa, A. (1996). «De la micro-histoire vers une anthropologie critique ». Jeux d'échelles. La micro-analyse à l'expérience. J. Revel. Paris, Gallimard-Le Seuil: 37-70.

Bessin M., C. Bidart and M. Grossetti. Bifurcations. Les sciences sociales face aux ruptures et à l'événement., Paris, La Découverte: 64-88.

Cefaï D. (2001) Expérience, culture et politique. Cefaï D. Cultures politiques, PUF, p.93-116. 
Cefaï, D. (2010) L'engagement Ethnographique. Paris: Ecole des Hautes Etudes en Sciences Sociales.

Dobry, M. (2010). Le politique dans ses états critiques : retour sur quelques aspects de l'hypothèse de continuité.

Fassin, D. (2008). « La politique des anthropologues ». L'Homme 185-186: 165-186.

Fentress J. \& Wickham C. (1994). Social Memory. Oxford, Cambridge: Blackwell.

Hartog F. et Jacques Revel J. (2001). Les usages politiques du passé. Introduction. Coll.Enquêtes, Paris, Éditions EHESS.

KayVaughan, M. (2001) «Transnational Processes and the Rise and Fall of the Mexican Cultural State». In Fragments of a Golden Age. The Politics of Culture in Mexico since 1940, edited by Gilbert Joseph, Anne Rubenstein and Eric Zolov. Durham: Duke University Press.

Lomnitz, C. (2001). Deep Mexico, Silent Mexico. An Anthropology of Nationalism. Minneapolis: University of Minneapolis Press.

Martínez-Vásquez V.R. (1990). Movimiento popular y política en Oaxaca : 19681986. Mexico: Consejo Nacional Para la Cultura y La Artes.

Nora P. (1984). Lieux de mémoire. La République. Paris: Gallimard.

Nuijten, M. (2004). « Between Fear and Fantasy. Governmentality and the Working of Power in Mexico ». Critique of Anthropology, 24(2), 209-230.

Passeron J.C. \& Revel J. (2005). Penser par cas. Paris: EHESS.

Recondo D. (2002). «État et coutumes électorales dans l'Oaxaca (Mexique) : réflexions sur les enjeux politiques du multiculturalisme». In Université Montesquieu-Bordeaux IV, 657. Bordeaux: Bordeaux IV.

Rubin, J. W. (2004). «Meanings and Mobilizations : A Cultural Politics Approach to Social Movements and States ». Latin American Research Review, 39 (3), 106-142.

Street S. (1997). «Los maestros y la democracia de los de abajo». In La democracia de los de abajo. Alonso J., Ramirez S.

J.M. (Eds), , La Jornada, UNAM, Colegio Electoral del Estado de Mexico, 115-145.

Yescas Martınez I., Zafra G. [1985] (2006). La Insurgencia Magisterial en Oaxaca 1980, 2nd Edition. Oaxaca : Serie Estudios Sociales Fonda Editorial IEEPO/IISUABJO. 